\title{
Evaluation of Questor urine screening system for bacteriuria and pyuria
}

\author{
M Stevens, C J Mitchell, S A Livsey, C A MacDonald
}

Department of Health Evaluation Unit, Public Health Laboratory, The Royal Infirmary, Leicester LE1 5WW

$M$ Stevens

C J Mitchell

$S$ A Livsey

Medical Devices

Directorate

Department of

Health, Russell

Square, London

C A MacDonald

Correspondence to:

M Stevens

Accepted for publication

7 April 1993

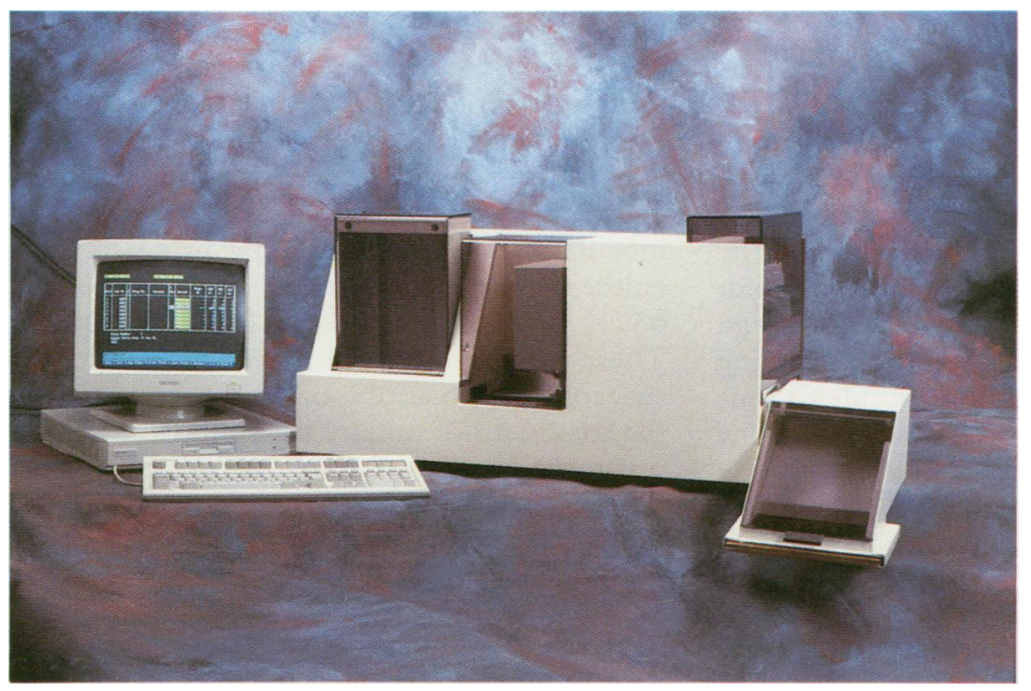

Figure 1 Complete Questor system with computer.

obtained from other screening systen using the same protocol. The system was easy to use and is a useful addition to the methods available for screening for bacteriuria.

$(\mathcal{C}$ Clin Pathol 1993;46:817-821)
Urinary infections are second only in prevalence to those of the upper respiratory tract. But the number of requests submitted to clinical microbiology laboratories for the microbiological examination of urine far exceeds the number of upper respiratory tract specimens. Bacteriuria may be chronic, persistent, and possibly influence structure and function outside the urinary tract. It has an important role in disease throughout life, from prematurity to hypertension and renal failure. ${ }^{1}$ Many methods for screening for bacteriuria have been described and reviewed, ${ }^{2}$ but there remains a need for an accurate, rapid, cost effective system for use in routine clinical laboratories. Questor (Difco Laboratories Ltd, East Molesey, Surrey) is an automated system which is designed to categorise and enumerate bacteria and other cells found in urine using particle counting technology.

An evaluation of the system was commissioned by the Medical Devices Directorate of the Department of Health and carried out at the Leicester Public Health Laboratory.

The system's performance was compared with a comprehensive reference method which included a variety of cultural techniques, microscopic examination, and clinical information. A full report of the evaluation has been produced. ${ }^{3}$

\section{Methods}

The Questor system (fig 1) is an automated single-channel impedance particle counter which uses a direct physical method to count and categorise particles. A $100 \mu 1$ aliquot of the urine to be tested is pipetted into one well of an eight-well disposable plastic tray which is then placed in the instrument. The following automatic process is then carried out. The specimen is diluted with a conductive electrolyte solution and offered to a particle counting probe (fig 2 ). There is a small orifice (A) in the glass end of the probe which also contains two platinum electrodes, one internal (B) and one external (C). An electrical field is established between the electrodes via the electrolyte in the orifice. The sample is drawn through the orifice under vacuum. As a particle is drawn through the orifice and enters the electrical field, it displaces its own volume of electrolyte, causing a change in the impedance of the electrical circuit. The changes are detected as pulses, the number of which indicate the quantity of particles present in the sample.

The height and area of the pulse is propor- 
Figure 2 Schematic representation of the Questor probe: $(A)$ orifice; (B) internal electrode; (C) external electrode; (D) movable insert showing direction of movement $(E)$

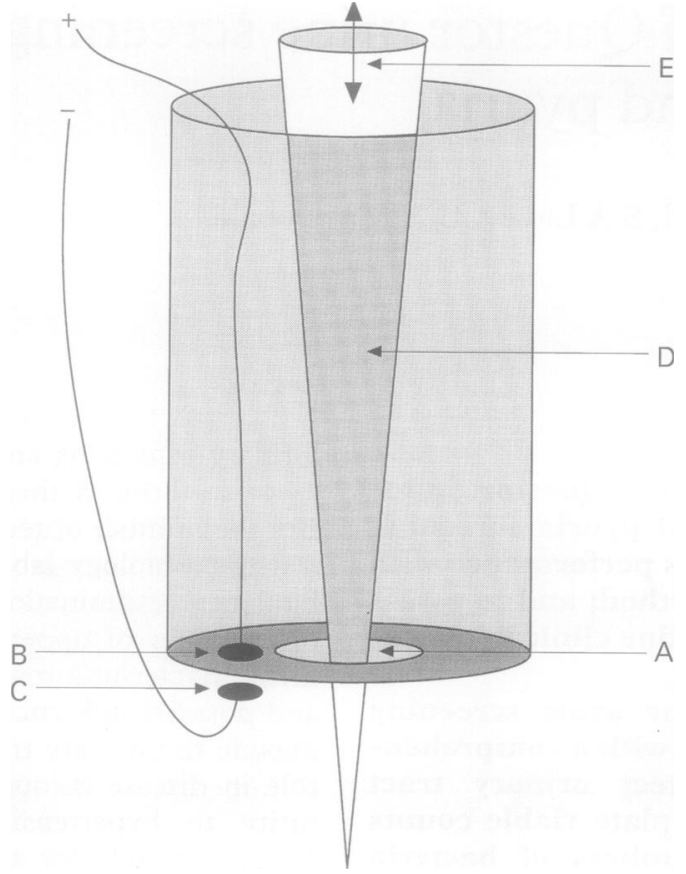

tional to the mean spherical volume of the particle. A histogram of the particles and their sizes is formed and analysed by the computer software to give enumeration of the particles of different size bands. In Questor, the volume of the orifice is varied by inserting a tapered spike (the insert) (D) into and out of the orifice (A). Thus the characteristics of the orifice can be optimised for the size of the particles of interest (from 0.5 to $40 \mu \mathrm{m}$ ). The analyser records the volume distributions for four different particle size ranges corresponding to organisms, erythrocytes, leucocytes and epithelial cells.

Threshold settings to define a positive result can be selected by the user. If the organism count is above the selected threshold-for example, $1.0 \times 10^{5}$-the interpretation given is "positive". If the leucocyte count is above the selected threshold- $\geqslant 50 / \mu \mathrm{l}$ - the interpretation given is "pos-white cell". If the erythrocyte count is above the selected threshold $-\geqslant 250 / \mu$ l-the interpretation given is "pos-red blood cell". The user has the option of choosing a threshold value for any combination of organisms, leucocytes, and erythrocytes above which results are interpreted as positive.

A previous evaluation of the Ramus urine screening system, ${ }^{4}$ which utilised particle counting, showed that the fixed, small size orifice used was prone to blocking. In the Questor system the manufacturers claim that, "putting the insert into the orifice forms an annulus. The flow pattern sets up a standing wave at the entrance of the annulus. This wave sweeps the large particles away from the annulus but allows the small particles through. This is called the 'filter effect', which greatly reduces the incidence of probe blockage."

Apart from the Questor system, the items required for testing urines are plastic trays and particle-free isotonic diluent (both of which are supplied by the manufacturer), a micropipette and tips, plastic waste disposal bags, and computer consumables: paper, ribbons and floppy disks.

\section{Evaluation methods}

The urine samples examined during the evaluation were random, unselected, consecutive specimens and were representative of a cross section of the types submitted to the routine clinical laboratory. A total of 1023 urine specimens were examined by Questor and by comprehensive comparative methods. Of the 1023, 1003 were received preserved in boric acid. The remaining 20 were unpreserved but were refrigerated and tested by Questor and the reference methods concurrently.

A pour-plate viable bacterial counting technique was used as the basis of the reference method. Urines were diluted in quarter strength Ringer solution to produce dilutions of $10^{2}$ and $10^{4}$. One millilitre of each dilution and of the undiluted urine was mixed with $20 \mathrm{ml}$ of sterile, molten Columbia agar base (Oxoid CM331) held at a temperature of $45^{\circ} \mathrm{C}$. Plates were incubated overnight at $37^{\circ} \mathrm{C}$ and colonies were counted. The range of dilutions was designed to allow bacterial numbers in the range 0 to $10^{7} \mathrm{cfu} / \mathrm{ml}$ to be counted. To facilitate the identification of organisms, a calibrated $1 \mu \mathrm{l}$ loop was used to seed urine on to one half of a $9 \mathrm{~cm}$ diameter agar plate containing cystine-lactoseelectrolyte deficient (CLED) medium (Oxoid CM301). Inoculated plates were incubated at $37^{\circ} \mathrm{C}$ for 18 hours. Isolates were identified using a variety of standard methods. ${ }^{5}$

Growths were considered to be clinically significant if a count of $\geqslant 10^{5} \mathrm{cfu} / \mathrm{ml}$ of no more than two types of organisms were grown or there was a predominant organism in a mixed culture. Counts between $10^{4}$ and $10^{5}$ $\mathrm{cfu} / \mathrm{ml}$ were considered to be clinically significant if they were in pure culture and the patient was receiving antimicrobial treatment, or the urine contained $\geqslant 10$ leucocytes $/ \mu 1$.

Cultures designed to detect and enumerate carboxyphilic and obligately anaerobic bacteria were prepared as follows: each urine sample was cultured, using a $1 / \mu$ l loop on to blood agar and heated blood agar plates. These were incubated anaerobically and in an atmosphere of $10 \% \mathrm{CO}_{2}$ in air, respectively, for 48 hours at $37^{\circ} \mathrm{C}$.

All urine samples were tested for the presence of antibacterial agents. A multipoint inoculator was used to apply urine to lawn cultures of Staphylococcus aureus (NCTC 6571) and Escherichia coli (NCTC 10418). These strains were chosen because of their susceptibility to antibiotics commonly used in the treatment of urinary tract infections. After overnight incubation, the presence of antibacterial agents was recorded where zones of inhibition occurred.

\section{Statistical analyses}

The results of individual tests from Questor and the reference method were categorised 
Table 1 Categories of results comparing reference and Questor methods

\begin{tabular}{lll}
\hline $\begin{array}{l}\text { Reference test } \\
\text { result }\end{array}$ & Questor result & \\
\cline { 2 - 3 } & Positive & Negative \\
\hline $\begin{array}{l}\text { Positive } \\
\text { Negative }\end{array}$ & True positive & False negative \\
\hline
\end{tabular}

(table 1). Using these categories, the sensitivity, specificity, and positive and negative predictive values were calculated as follows ${ }^{6}$ :

Sensitivity (\%)

$\frac{\text { True positives }}{\text { True positives }+ \text { false negatives }}$

Specificity (\%)

True negatives

True negatives + false positives

Positive predictive value (\%)

True positives

True positives + false positives

Negative predictive value (\%)

True negatives

True negatives + false negatives

The performance of Questor was calculated by using varying threshold settings for the organism counts between $1.0 \times 10^{4}$ to $1.1 \times 10^{5}$ in steps of $0.1 \mathrm{log}$, together with leucocyte counts of $20,30,40$ and 50 per $\mu$ 1 and an erythrocyte count of $\geqslant 250$ (table 2). From the many possible combinations of thresholds, the optimal performance was calculated using the method of Koenig et al. ${ }^{7}$ In this method sensitivity is weighted more heavily than specificity, because the consequences of reporting a false negative result are potentially more serious than those of a

Table 2 Performance of Questor at different positive thresholds

\begin{tabular}{|c|c|c|c|}
\hline $\begin{array}{l}\text { Questor } \\
\text { organisms }\end{array}$ & $\begin{array}{l}\text { Sensitivity } \\
(\%)\end{array}$ & $\begin{array}{l}\text { Specificity } \\
(\%)\end{array}$ & $\begin{array}{l}\text { Performance } \\
\text { index }\end{array}$ \\
\hline \multicolumn{4}{|l|}{ Leucocytes $50 \mu \mathrm{l}$} \\
\hline $5.0 \times 10^{4}$ & $93 \cdot 2$ & $59 \cdot 0$ & $83 \cdot 0$ \\
\hline $6.0 \times 10^{4}$ & $93 \cdot 2$ & $65 \cdot 3$ & $84 \cdot 8$ \\
\hline $7.0 \times 10^{4}$ & $92 \cdot 7$ & $71 \cdot 3$ & $86 \cdot 3$ \\
\hline $8.0 \times 10^{4}$ & $91 \cdot 0$ & $75 \cdot 8$ & $86 \cdot 4$ \\
\hline $9.0 \times 10^{4}$ & $89 \cdot 8$ & $77 \cdot 7$ & $86 \cdot 2$ \\
\hline $1.0 \times 10^{5}$ & $89 \cdot 3$ & $78 \cdot 4$ & $86 \cdot 0$ \\
\hline $1 \cdot 1 \times 10^{5}$ & $88 \cdot 7$ & $79 \cdot 3$ & $86 \cdot 9$ \\
\hline \multicolumn{4}{|l|}{ Leucocytes $40 \mu l$} \\
\hline $5.0 \times 10^{4}$ & $93 \cdot 2$ & $58 \cdot 0$ & $82 \cdot 7$ \\
\hline $6.0 \times 10^{4}$ & $93 \cdot 2$ & $64 \cdot 2$ & $84 \cdot 5$ \\
\hline $7.0 \times 10^{4}$ & $93 \cdot 2$ & $70 \cdot 0$ & $86 \cdot 3$ \\
\hline $8.0 \times 10^{4}$ & $92 \cdot 7$ & $74 \cdot 0$ & $87 \cdot 1$ \\
\hline $9.0 \times 10^{4}$ & 91.5 & $75 \cdot 9$ & 86.9 \\
\hline $1.0 \times 10^{5}$ & 91.5 & $76 \cdot 5$ & $87 \cdot 0$ \\
\hline $1.1 \times 10^{5}$ & 91.0 & $77 \cdot 2$ & $86 \cdot 8$ \\
\hline \multicolumn{4}{|l|}{ Leucocytes $30 \mu l$} \\
\hline $5.0 \times 10^{4}$ & $93 \cdot 8$ & $57 \cdot 1$ & $82 \cdot 8$ \\
\hline $6.0 \times 10^{4}$ & $93 \cdot 8$ & $63 \cdot 0$ & $84 \cdot 6$ \\
\hline $7.0 \times 10^{4}$ & $93 \cdot 8$ & $68 \cdot 3$ & $86 \cdot 2$ \\
\hline $8.0 \times 10^{4}$ & $93 \cdot 2$ & $71 \cdot 8$ & $86 \cdot 8$ \\
\hline $9.0 \times 10^{4}$ & $92 \cdot 1$ & $74 \cdot 0$ & $86 \cdot 7$ \\
\hline $1.0 \times 10^{5}$ & $92 \cdot 1$ & $74 \cdot 0$ & $86 \cdot 7$ \\
\hline $1.1 \times 10^{5}$ & 91.5 & $74 \cdot 5$ & $86 \cdot 4$ \\
\hline \multicolumn{4}{|l|}{ Leucocytes $20 \mu \mathrm{l}$} \\
\hline $5.0 \times 10^{4}$ & $94 \cdot 9$ & $54 \cdot 6$ & $82 \cdot 8$ \\
\hline $6.0 \times 10^{4}$ & $94 \cdot 9$ & $59 \cdot 7$ & $84 \cdot 4$ \\
\hline $7.0 \times 10^{4}$ & $94 \cdot 9$ & $64 \cdot 1$ & $85 \cdot 7$ \\
\hline $8.0 \times 10^{4}$ & $94 \cdot 4$ & $67 \cdot 3$ & $86 \cdot 2$ \\
\hline $9.0 \times 10^{4}$ & $93 \cdot 8$ & $68 \cdot 4$ & $86 \cdot 2$ \\
\hline $1.0 \times 10^{5}$ & $93 \cdot 8$ & $68 \cdot 9$ & $86 \cdot 3$ \\
\hline $1.1 \times 10^{5}$ & $93 \cdot 8$ & $69 \cdot 2$ & $86 \cdot 4$ \\
\hline
\end{tabular}

false positive result. The optimal performance was determined by calculating an index that emphasised sensitivity more than specificity in a ratio of $70: 30-70 \%$ of the sensitivity was added to $30 \%$ of the specificity at each threshold combination (table 2). The 70:30 ratio is arbitrary; small changes in the ratio do not alter the optimal thresholds.

\section{Results}

Of the 1023 urine samples tested, $225(22 \%)$ contained $\geqslant 10^{5} \mathrm{cfu} / \mathrm{ml}$ of bacteria as determined by the pour-plate counts. Of these 225,167 were considered to be clinically significant growths. An additional 10 specimens contained between $10^{4}$ and $10^{5} \mathrm{cfu} / \mathrm{ml}$ and were considered to be clinically significant. Because the growth was pure, it was associated with the presence of 10 or more leucocytes and the clinical information indicated an infection. There were therefore a total of 177 significant growths.

Using the method of Koenig et $a l^{7}$; the optimal performance of Questor was with the following thresholds: bacteria $8.0 \times 10^{4}$, or leucocytes $40 / \mu \mathrm{l}$, or erythrocytes $250 / \mu \mathrm{l}$. The performance achieved at these thresholds is shown in table 3.

Of the 13 false negative results 10 grew pure cultures of $E$ coli, all of which had less than 10 leucocytes $/ \mu 1$. One specimen grew Enterococcus faecalis and another Proteus mirabilis, associated with leucocyte counts of $1 / \mu 1$ and $6 / \mu 1$, respectively. The clinical significance of these growths may be questioned. The remaining false negative grew $E$ coli and had a leucocyte count of $22 / \mu \mathrm{l}$.

Questor assigned the following interpretations to the false positive results: $(n=$ number of samples in category): "positive" 109, "pos white cell" 84, and "pos-red blood cell" 27.

The leucocyte counts of the false positive results determined by microscopy is shown in table 4. Questor was thus able to detect a large number of leucocytes $(\geqslant 50)$ in almost $50 \%$ of the false positive results.

Of the 220 false positive results, 86 (39\%) contained anaerobic or carboxyphilic organisms and a further 74 (34\%) contained a mixed bacterial population. Thus in $73 \%$ of

Table 3 Optimal performance of Questor

\begin{tabular}{lc}
\hline True positives & 164 \\
True negatives & 626 \\
False positives & 220 \\
False negatives & 13 \\
Sensitivity & $92 \cdot 7 \%$ \\
Specificity & $74 \cdot 0 \%$ \\
Positive predictive value & $42 \cdot 7 \%$ \\
Negative predictive value & $98 \cdot 0 \%$ \\
\hline
\end{tabular}

Table 4 False positive samples containing leucocytes

\begin{tabular}{lc}
\hline Number of leucocytes & Number of samples \\
\hline $0-9$ & 72 \\
$10-19$ & 17 \\
$20-29$ & 5 \\
$30-39$ & 8 \\
$40-49$ & 9 \\
$50-99$ & 21 \\
$\geqslant 100$ & 88 \\
\hline
\end{tabular}


the false positive results, organisms were present in numbers greater than $10^{5} \mathrm{cfu} / \mathrm{ml}$. Antibiotic substances were detected in a significantly higher percentage (47 of 220 , or $21 \%$ ) of the false positive samples compared with $147 / 1023(14 \%)$ of all the samples tested $(\mathrm{p}=0.001)$.

Questor assigned the following interpretation to the true positive results: $(n=$ number of samples in category): "positive" 105, "poswhite cells" 48, and "pos-red blood cells" 10 . Comparisons between the Questor leucocyte, erythrocyte, and epithelial cells counts and microscopy are given in tables 5, 6, and 7 respectively.

\section{Discussion}

The Questor system was more sensitive and specific than other bacteriuria screening systems evaluated using the same protocol. ${ }^{489}$

The results showed that the system was able selectively to detect bacteria, leucocytes, erythrocytes and epithelial cells. The bacterial counts given were accurate, except below $1.0 \times 10^{4}$ and above $1.1 \times 10^{5}$, and the evaluators suggest that as part of the installation process for individual laboratories, the software should be configured so that results outside these figures should read $<1.0 \times 10^{4}$ and $>1.0 \times 10^{5}$, respectively.

Although Questor recorded a false positive result for 220 specimens, many of these could be accounted for by the presence of anaerobic or carboxyphilic organisms, or the presence of antimicrobial substances and therefore the possibility of the presence of non-viable bacteria.

A total of 109 of the 220 false positive results contained high numbers of leucocytes

Table 5 Leucocyte counts, Questor compared with microscopy Questor white cell count (per $\mu l$ )

\begin{tabular}{lrrrrr}
\hline Microscopy & $0-9$ & $10-29$ & $30-49$ & $\geqslant 50$ & Totals \\
\hline $0-9$ & 486 & 129 & 29 & 14 & 658 \\
$10-29$ & 14 & 44 & 9 & 16 & 83 \\
$30-49$ & 0 & 13 & 18 & 13 & 44 \\
$\geqslant 50$ & 1 & 8 & 8 & 221 & 238 \\
Totals & 501 & 194 & 64 & 264 & 1023 \\
\hline
\end{tabular}

Table 6 Erythrocyte counts, Questor compared with microscopy Questor red blood cell count (per $\mu l)$

\begin{tabular}{lrrrrr}
\hline Microscopy & $0-9$ & $10-29$ & $30-49$ & $\geqslant 50$ & Totals \\
\hline $0-9$ & 524 & 141 & 30 & 37 & 732 \\
$10-29$ & 17 & 43 & 13 & 5 & 78 \\
$30-49$ & 1 & 10 & 9 & 11 & 31 \\
$\geqslant 50$ & 6 & 23 & 28 & 125 & 182 \\
Totals & 548 & 217 & 80 & 155 & 1023 \\
\hline
\end{tabular}

Table 7 Epithelial cells, Questor compared with microscopy Questor epithelial cell count (per $\mu l$ )

\begin{tabular}{lrcccc}
\hline Microscopy & $0-9$ & $10-29$ & $30-49$ & $\geqslant 50$ & Totals \\
\hline $0-9$ & 687 & 34 & 1 & 1 & 723 \\
$10-29$ & 84 & 57 & 3 & 1 & 145 \\
$30-49$ & 9 & 34 & 1 & 0 & 44 \\
T50 & 3 & 16 & 14 & 22 & 55 \\
Totals & 783 & 141 & 19 & 24 & 967 \\
\hline
\end{tabular}

*Epithelial cells were not recorded by Questor for the remaining 56 specimens, due to the presence of an excess number of leucocytes. and although no organisms were detected in these samples, these high leucocyte counts have diagnostic value.

Questor has a tendency to give higher leucocyte and erythrocyte counts than is found by microscopy (tables 5 and 6 ).

Individual laboratories using Questor have the option of choosing any combination of different positive threshold levels for organisms, leucocytes, and erythrocytes. Changing these thresholds will alter the performance, increasing the sensitivity and lowering the specificity or vice versa. This may be important when testing urine samples from particular groups of patients. In a patient population with a low incidence of urinary tract infection the thresholds could be changed to increase the specificity; in a symptomatic group, a high sensitivity is required. It is recommended that each user laboratory should calibrate the system by comparing the results obtained with those of the best possible comparative method.

Because the operator involvement in the analytical process consists only of pipetting $100 \mu \mathrm{l}$ of urine sample into a tray and entering the sample number into the computer system, with no interpretive steps, a medical laboratory assistant can be used to operate the system.

In this evaluation it was possible for a medical laboratory assistant to operate the Questor system and to process 230 urine samples a day. As Questor takes about 1 minute to process one sample, in theory it would be possible for the system to test over 400 samples in a normal working day. This, however, would rely on specimens being received at regular intervals and therefore available for testing as a continuous workload throughout the day. In practice this did not occur, with most samples being received in mid-afternoon. Questor is a system designed to screen urines, allowing a negative result to be sent from the laboratory in most cases on the same day as specimen receipt.

This could have some beneficial impact on patient care, although this would depend greatly on both the speed at which the specimen is sent to the laboratory after voiding and how quickly the report is sent to the requesting physician. A benefit to the laboratory would be that the negative specimens would not be cultured and therefore not require the preparation and interpretation of culture plates by laboratory staff. Specimens recorded as positive by Questor would require culture, to identify causative organisms, to provide cultures for antimicrobial susceptibility tests, and to detect mixed, possibly insignificant growths.

The list price capital cost of the Questor system is $£ 39950$. The list price costs of the consumables required, diluent, and trays total $£ 0.17$ a sample. The WELCAN workload system, when applied to all procedures associated with urine screening by Questor and a routine microscopical and cultural method, showed a saving of staff time of $31 \%$ when using the automated method. 
The system was simple to use, was liked by laboratory staff, and had excellent, userfriendly software. The ability of the system to provide an accurate rapid, result, the savings in staff time taken to process samples make the Questor system an important and useful addition to the methods available for screening for bacteriuria.

This evaluation was supported financially by the Medical Devices Directorate, Department of Health.

1 Kass EH. Pyelonephritis and bacteriuria: a major problem in preventative medicine. Ann Intern Med 1962;56: 46-53.

2 Stevens M. Screening urines for bacteriuria. Med Lab Sci
$1989 ; 46: 194-206$.

3 Medical Devices Directorate. An evaluation of the Questor urine screening system. Report MDD/92/48. London: Department of Health, 199

4 Medical Devices Directorate. An evaluation of the Orbec Ramus 256. Report STD/88/13. London: Department of Health, 1988.

5 Cowan E Steel's Manual for the Identification of Medical Bacteria. 2nd Edn, Cambridge: Cambridge University Press, 1975.

6 Galen RS, Gambino SR. Beyond Normality: The Predictive Value and Efficiency of Medical Diagnoses. New York: Vohn Wiley and Sons, 1975 .

John Wiley and Sons, 1975.

Koenig C, Tick LJ, Hanna BA. Analyses of the UTIscreen bioluminescence tests for bacteriuria. F Clin Microbiol 1992;30: $342-5$

8 Medical Devices Directorate. An evaluation of the Autotrak urine screening system. Report STD/88/28. London Department of Health, 1988 .

9 Medical Devices Directorate. An evaluation of the Sensititre LeukoBact urine screen. Report MDD/92/03. London: Department of Health, 1992. 